\title{
EXPERIMENTS ON HYPERSONIC BOUNDARY LAYER TRANSITION ON BLUNT CONES WITH ACOUSTIC-ABSORPTION COATING
}

\author{
A. Shiplyuk ${ }^{1}$, S. Lukashevich ${ }^{1}$, D. Bountin ${ }^{1}$, A. Maslov ${ }^{1}$, \\ and H. Knaus ${ }^{2}$ \\ ${ }^{1}$ Khristianovich Institute of Theoretical and Applied Mechanics \\ Siberian Branch of the Russian Academy of Sciences \\ Novosibirsk, Russia \\ ${ }^{2}$ IAG, Stuttgart University \\ Stuttgart, Germany
}

The laminar-turbulent transition is studied experimentally on a cone with an acoustic-absorption coating and with different nose bluntness in a high-speed flow. The acoustic-absorption coating is a felt metal sheet with a random microstructure. Experiments were carried out on a 1-meter length 7 degree cone at free-stream Mach number $\mathrm{M}=8$ and zero angle of attack. Locations of the laminar-turbulent transition are detected using heat flux distributions registered by calorimeter sensors. In addition, boundary layer pulsations are measured by means of ultrafast heat flux sensors. It is shown that the laminar-turbulent transition is caused by the second-mode instability, and the laminar run extends as the bluntness is increased. The porous coating effectively suppresses this instability for all tested bluntness values and 1.3-1.85 times extends the laminar run.

\section{NOMENCLATURE}

$\mathrm{M}_{\infty} \quad$ free-stream Mach number

$R \quad$ nose bluntness radius

$\mathrm{Re}_{\mathrm{tr}} \quad$ Reynolds number of laminar-turbulent transition beginning

$\operatorname{Re}_{1 \infty} \quad$ free stream unit Reynolds number, $\mathrm{m}^{-1}$

St Stanton number

$X \quad$ longitudinal coordinate measured along the cone surface, $\mathrm{mm}$

This is an Open Access article distributed under the terms of the Creative Commons Attribution-Noncommercial License 3.0, which permits unrestricted use, distribution, and reproduction in any noncommercial medium, provided the original work is properly cited. 

$X_{\text {por }} \quad$ longitudinal coordinate of laminar-turbulent transition beginning on porous surface
$X_{\text {sol }} \quad$ longitudinal coordinate of laminar-turbulent transition beginning on solid surface
$X_{\mathrm{tr}} \quad$ longitudinal coordinate of laminar-turbulent transition beginning
$\theta \quad$ the angle of model rotation

\section{INTRODUCTION}

At hypersonic velocities, the laminar-turbulent transition in boundary layers is caused by the 2nd-mode disturbances which are self-acoustic oscillations of these boundary layers. In $[1,2]$, a new concept of controlling the hypersonic laminar boundary layers with ultrasonic-absorbing coatings (UAC) was proposed. The concept stems from the fact that the hypersonic boundary layer behaves as an acoustic waveguide [2] wherein nonstable 2nd-model disturbances propagate. Malmuth et al. [1] suggested that the absorption of acoustic energy by $\mathrm{UAC}$ can stabilize the 2nd mode of the boundary layer disturbance. Analysis of stability $[1,2]$ showed that the UAC is able to reduce essentially the degree of the 2nd-mode amplification and to increase in several times the laminar part length.

This concept was experimentally checked in California Institute of Technology (Caltech) in the wind tunnel GALCIT T5 at Mach numbers $\mathrm{M}=5-6$ on a sharp cone [3]. The porous coating for absorbing the boundary layer disturbances presented a plate with equidistant cylindrical blind holes. Experiments showed that the UAC could effectively delay the transition which proved the theoretical concept. It was also demonstrated that the holes could influence the boundary layer as the distributed surface roughness and resulted in earlier transition.

Application of the surfaces with chaotic porous microstructure for passive control of laminar flows in hypersonic aircrafts seems promising because of the low price of these surfaces. Experimental investigations of developing natural disturbances and artificial wave packets on the surface with the chaotic porous microstructure were performed in ITAM at Mach number $\mathrm{M}_{\infty}=6$ and heat-insulated wall conditions [4]. The material consisted of thin stainless-steel wires, disposed chaotically and sintered to each other. It was shown that the surface with the chaotic porous microstructure stabilizes high-frequency 2ndmode disturbances and destabilizes relatively low-frequency 1st-mode disturbances.

Experimental investigations of the laminar-turbulent transition on the sharp cone with the passive chaotically-porous coating were carried out in hypersonic wind tunnel AT-303 ITAM at Mach number $\mathrm{M}_{\infty}=12[5]$ and zero angle of 
attack. It was demonstrated that the passive porous coating with the chaotic microstructure considerably delays the transition. The beginning of the transition was also calculated for the cases of solid and porous surfaces using the $e^{N_{\text {}}}$ method and semiempirical method of definition of acoustic properties of porous coatings with chaotic microstructure, regarding the rarefaction effect. Calculation results showed satisfactory agreement with the experimental results. The present authors pioneered to show that the chaotic porous coating, very similar in its structure to a fibrous ceramic heat-insulation material, can be used to control hypersonic laminar flows.

Since real hypersonic vehicles have a blunted leading edge, this work presents an experimental investigation of the effect of the ultrasonic-absorbing coating on the laminar-turbulent transition on a cone with various bluntnesses of the nose part, at zero angle of attack, and Mach number $\mathrm{M}_{\infty}=8$.

\section{MODEL}

The experimental model was designed in a way to provide the possibility to measure the transition position both on the porous and solid model walls in one run. The model presents a cone with the half-angle $7^{\circ}$. Half surface along the generatrix is coated with the porous coating Bekipor ${ }^{\circledR}$ made by Bekaert Company (USA). Total length of the cone together with the sharp nose is $1039 \mathrm{~mm}$. The porous coating begins $70 \mathrm{~mm}$ away and consists of thin stainless-steel wires $30 \mu \mathrm{m}$ in diameter, chaotically disposed and sintered to each other. The coating has the porosity of $75 \%$ and total thickness $0.75 \pm 0.1 \mathrm{~mm}$. To study the influence of the nose part bluntness on the transition, exchangeable nose parts with spherical bluntness $R=0,1,2,4$, and $8 \mathrm{~mm}$ were used. Below, these nose parts are named $\mathrm{R} 0, \mathrm{R} 1, \mathrm{R} 2, \mathrm{R} 4$, and $\mathrm{R} 8$, respectively.

The position of the laminar-turbulent transition was found from the results of heat flux measurements. The heat flux was measured by calorimetric gages. Fifty-eight gages were installed for simultaneous measurement of the heat-flux distributions over the porous and solid surfaces. The measurement system containing 55 independent analog-to-digital converters (ADC) with preamplifiers and low frequency filters was used to gather the data. Signals from each gage were digitized with the frequency of $5 \mathrm{kHz}$.

To obtain physical values of the heat fluxes, the calorimetric gages were calibrated. A thermal gun with the known heat flux $\left(q=0.127 \mathrm{MW} / \mathrm{m}^{2}\right)$ was used as a heat source. Maximum deviation of sensitivity factors from the average value did not exceed $\pm 2 \%$ for one gage.

Pulse characteristics of the boundary layer were measured with two atomic layer thermopiles (ALTP) gages [6] located on the porous and solid surfaces at the distance of $x=910 \mathrm{~mm}$ from the sharp nose. Signals from each gage were digitized with the frequency of $1 \mathrm{MHz}$. 


\section{EXPERIMENTAL RESULTS}

The measurements are performed in hypersonic wind tunnel of adiabatic compression AT-303 in ITAM SB RAS at free-stream Mach number $\mathrm{M}_{\infty}=8$. To produce the hypersonic flow, a contoured nozzle with the output diameter $D=400 \mathrm{~mm}$ is used.

\subsection{Flow Parameters}

The model is installed in the test section in a way to match the model axis and nozzle axis; the model position is checked with the aid of measuring tools. Slight deviations of the model axis setting angle are still possible. A small slipping angle of the cone in the horizontal plane would not influence the results of these investigations, since the heat-flux gages are on the top and bottom surfaces of the model, and their readings are compared to each other. Thus, here, only the effect of the angle of attack in the vertical plane should be considered. To evaluate this effect, the experiment is repeated twice for each combination of flow parameters with two orientations of the porous surface (top and bottom). For this, the model is rotated through $180^{\circ}$ around its axis; $\theta$ is the angle of model rotation; $\theta=0^{\circ}$ corresponds to the position of the porous coating on the cone bottom.

The experiments are carried out at Mach number $\mathrm{M}_{\infty}=8$ and three unit Reynolds numbers $\operatorname{Re}_{1 \infty}=4.4 \cdot 10^{6}, 5.2 \cdot 10^{6}$, and $6.5 \cdot 10^{6} \mathrm{~m}^{-1}$. During each run, there is a time interval from 30 to $50 \mathrm{~ms}$ with almost constant flow parameters. Calorimetric gages readings are processed and heat fluxes are calculated within these gaps.

\subsection{Heat Fluxes Distributions over Solid and Porous Surfaces}

Heat fluxes distributions over the solid and porous surfaces are constructed by the processed results of calorimetric gages readings. As an example, Fig. 1 shows the heat fluxes distributions for the nose R2 on the solid and porous surfaces, respectively, for the unit Reynolds number $\operatorname{Re}_{1 \infty}=6.5 \cdot 10^{6} \mathrm{~m}^{-1}$. Coordinate $X$ begins on the cone nose and coincides with the cone axis of symmetry. Obtained results show good agreement between the heat-fluxes distributions obtained for 2 orientations of the porous surface: $\theta=0^{\circ}$ and $180^{\circ}$. It means that the angle of attack approaches zero and free stream is uniform though, of course, there are small differences in heat fluxes values at various orientations of the porous surface. Heat fluxes values on the cone bottom part are on average $12 \%$ higher than on the top one and $15 \%$ higher than on the porous surface. Later on, the position of the laminar-turbulent transition $X_{\mathrm{tr}}$ is found from the averaging of 


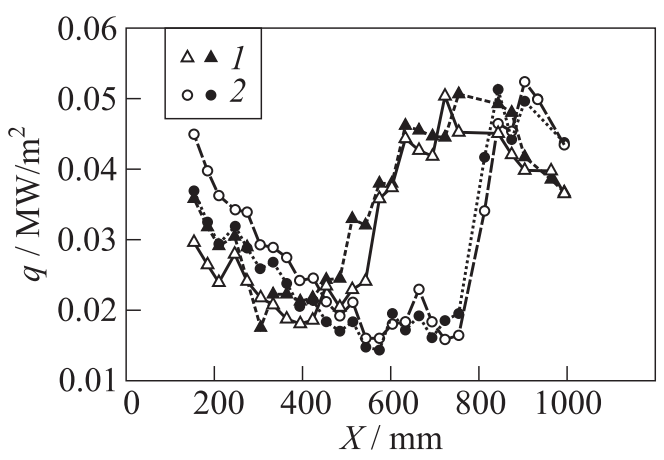

Figure 1 Heat fluxes distributions for the nose $\mathrm{R} 2$ and $\mathrm{Re}_{1 \infty}=6.5 \cdot 10^{6} \mathrm{~m}^{-1}: 1-$ solid surface; and 2 - porous surface. Empty signs refer to $\theta=180^{\circ}$ and filled signs to $\theta=0^{\circ}$

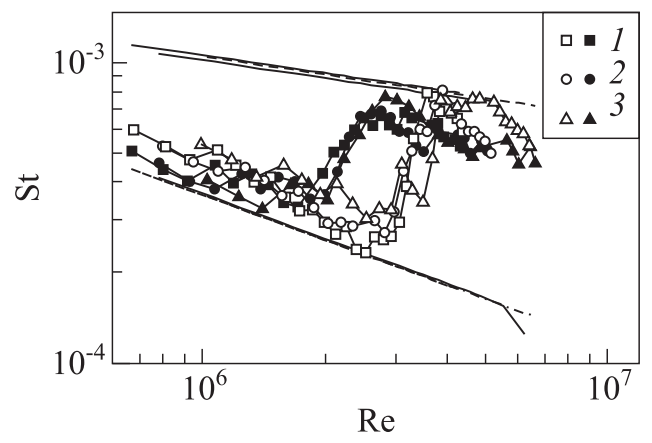

Figure 2 Heat fluxes distributions on the top part of the model for the nose R1: $1-\operatorname{Re}_{1 \infty}=4.4 \cdot 10^{6} \mathrm{~m}^{-1} ; 2-5.2 \cdot 10^{6}$; and $3-\mathrm{Re}_{1 \infty}=6.5 \cdot 10^{6} \mathrm{~m}^{-1}$. Empty symbols refer to porous surface and filled symbols to solid surface; dashed line refers to calculation of the turbulent boundary layer and dash-dotted to calculation of the laminar boundary layer

the transition coordinates, obtained in 2 runs at the same Reynolds numbers, at 2 orientations of the porous surface (top and bottom).

Figure 2 shows an example of heat fluxes distribution in the dimensionless form, for the nose R1. Graphs show Stanton numbers distribution over the top part of the cones vs. the free-stream unit Reynolds number $\operatorname{Re}_{1 \infty}$. Figure 2 also presents the results of heat fluxes calculation on the sharp cone for the laminar and turbulent boundary layers of an ideal gas. The measured heat fluxes demonstrate the typical pattern of cone heating in a hypersonic flow. In the laminar-flow region, the heat flux decreases as $X$ rises. In the transition area, dramatic (2-4 times) amplification of the heat flux is observed, then it reduces 
again in the turbulent region. In the configuration with the bluntness radius $\mathrm{R} 4$, there is no transition on the porous surface. It is seen from the graphs that the heat fluxes value in the laminar part of the flow is, in average, 14\%-21\% bigger on the porous part than on the solid one.

\subsection{Determination of the Laminar-Turbulent Transition Position}

Positions of the laminar-turbulent transition are determined from the obtained distributions of the heat fluxes. To define the position of the transition beginning and end, it is assumed that the transition beginning corresponds to the point with the minimum heat-flux value and the transition end corresponds to the point with the maximum heat-flux value.

Obtained dependencies of the Reynolds number of the transition beginning $\mathrm{Re}_{\mathrm{tr}}$ on the unit free-stream Reynolds number are shown in Fig. 3. It is seen from the graphs that $\mathrm{Re}_{\mathrm{tr}}$ is almost constant for small radii of the nose part bluntness ( $\mathrm{R} 0$ and $\mathrm{R} 1$ ) and rises with rising $\mathrm{Re}_{1 \infty}$ for big bluntness radii. It is also evident that the length of the laminar part is bigger on the porous surface than on the solid one and the laminar part increases at bigger bluntness radius.

To understand the efficiency of the porous coating for various bluntness values, Fig. 4 shows the ratios of the transition beginning coordinate $X_{\text {por }}$ on the porous surface to $X_{\text {sol }}$ on the solid surface regarding the nose part bluntness radius. For each bluntness radius, there are three dots corresponding to three free-stream Reynolds numbers presented on the graph. The dots with arrows show that under these conditions, the transition took place beyond the cone. In spite of some error in the experimental results, it is evident that the coating with

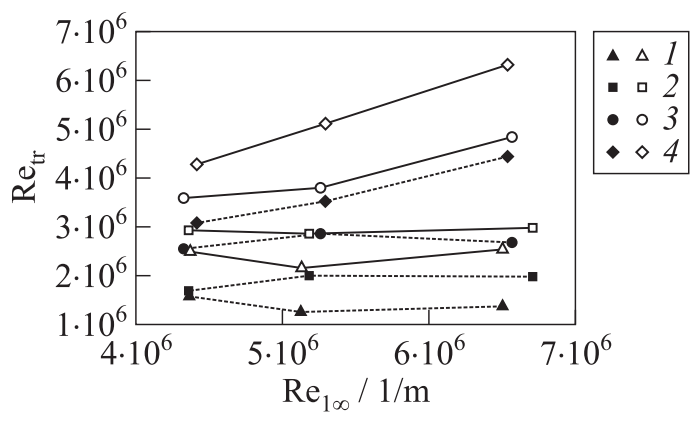

Figure 3 Dependence $\mathrm{Re}_{\mathrm{tr}}$ on $\mathrm{Re}_{1 \infty}$ for each experiment: $1-R_{0} ; 2-R_{1} ; 3-$ $R_{2}$; and $4-R_{4}$. Filled signs refer to solid surface and empty signs to porous surface 


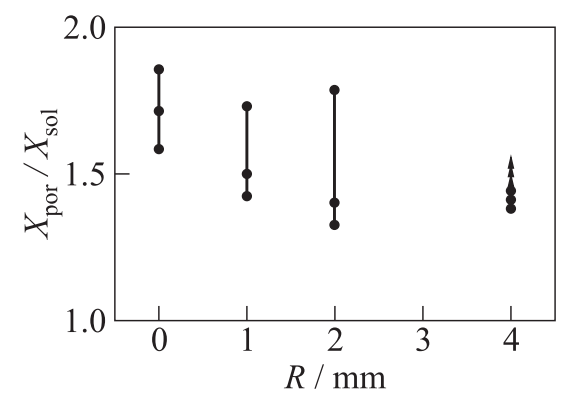

Figure 4 Ratio $X_{\text {por }}$ to $X_{\text {sol }}$ vs. $R$

the chaotic porosity increases effectively (1.3-1.85) the length of the laminar part in each experiment for the sharp and blunted cones.

\subsection{Heat Flux Pulsations}

Figure $5 a$ presents the spectra of heat fluxes pulsations for Reynolds number $\mathrm{Re}_{1}=4.4 \cdot 10^{6} \mathrm{~m}^{-1}$. For the sharp nose, on both surfaces, the spectra are turbulent (see Fig. 5, R0); however, the form of the spectra differs in the solid and porous cases. The difference is apparently caused by the effective suppression of the high-frequency disturbances of the boundary layer by the porous surface.

For the nose bluntness R2, the gage on the solid surface shows the turbulent state of the boundary layer, which fits well with the heat-fluxes distribution along the cone generatrix which shows that the transition takes place upstream from the gage. For R4 the spectrum shows that the boundary layer is in the transition state. A peak on the frequency $f \approx 140 \mathrm{kHz}$ stands against the spectrum. The frequency of this peak depends on the Reynolds number and shifts somehow at the model rotation through $180^{\circ}$, i. e., it is sensitive to slight variations of the angle of attack. Such a behavior is typical for the 2 nd mode, since its frequency depends on the boundary layer thickness. Thus, this peak is most likely to be the 2nd mode disturbances. For the biggest bluntness R8, the spectrum is laminar. In addition to the peak associated with the 2 nd mode $(f \approx 125 \mathrm{kHz}$, the frequency has changed due to the varying boundary layer thickness), there is another wide peak in the spectrum, in the low-frequency area. The origin of this peak is vague. Low-frequency pulsations appearance may be caused by the acoustic noise effect in the free stream.

On the porous surface for R1, the spectrum is turbulent (see Fig. 2, curve 1). In contrast to the solid surface for R2, on the porous surface the spectrum demonstrates that the boundary layer is in the transition state. It means that the porous surface shifts the transition downstream. The peak at the frequency 


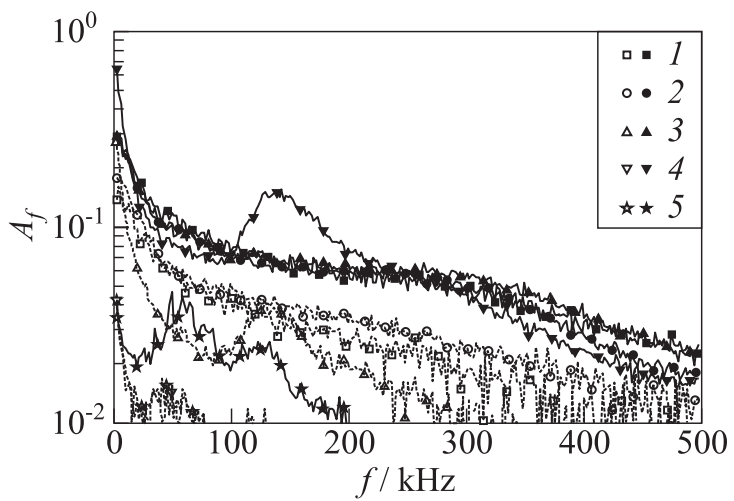

(a)

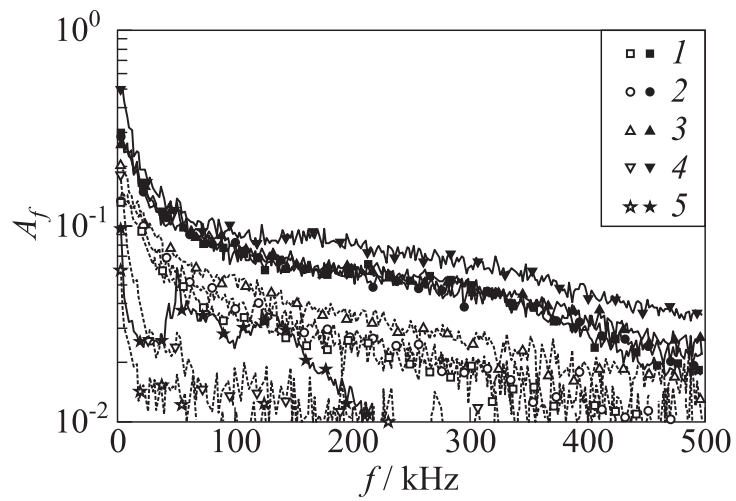

(b)

Figure 5 Spectra of heat fluxes pulsations for $\operatorname{Re}_{1}=4.4 \cdot 10^{6} \mathrm{~m}^{-1}(a)$ and $\operatorname{Re}_{1}$ $=6.5 \cdot 10^{6} \mathrm{~m}^{-1}(b)$ at various bluntnesses of the model nose: filled symbols - solid; empty symbols - porous; $1-R_{0} ; 2-R_{1} ; 3-R_{2} ; 4-R_{4}$; and $5-R_{8}$

$f \approx 130 \mathrm{kHz}$ is most likely the 2nd-model disturbances. For the bluntensses $\mathrm{R} 4$ and $\mathrm{R} 8$ on the porous surface, the boundary layer is laminar in the gage cross section and the spectra show almost total lack of pulsations. At the same time, on the solid surface in the laminar region, the pulsations are observed up to $200 \mathrm{kHz}$ (see Fig. $5 a, \mathrm{R} 8$ ). Lack of disturbances on the porous surface may be related with the fact that an entropy layer forms owing to the model nose bluntness; in a certain section, this layer attaches to the boundary layer. The point of attachment can be the source of boundary layer sensitivity to the acoustic noise existing in the free stream. Since the porous surface suppresses acoustic disturbances, the level of pulsations is much lower on it than on the solid surface. 
The same spectra but for higher unit Reynolds number are shown in Fig. $5 b$. Before the bluntness $R_{2}$, the boundary layer on the solid surface is turbulent, which correlates with the heat-fluxes distribution. For R4, the boundary layer is in the transition state, and because of the intermittency effect, the pulsation level turned out to be slightly higher than for smaller bluntness. With the bluntness R8, the boundary layer is laminar on both sides, which is seen by the pulsation spectra. Again two peaks are seen in the pulsation spectrum, similarly to $\operatorname{Re}_{1}=4.4 \cdot 10^{6} \mathrm{~m}^{-1}$.

\section{CONCLUDING REMARKS}

The laminar-turbulent transitions of the hypersonic boundary layer is studied experimentally on the cone with the porous coating and different bluntnesses of the nose part for three Reynolds numbers, to determine the effect of the ultrasonic-absorbing coating on the transition position at zero angle of attack. The experiment is performed on a cone of 1 -meter length with the half-angle $7^{\circ}$ in hypersonic wind tunnel AT-303, ITAM, at free-stream Mach number $\mathrm{M}_{\infty}=8$. The ultrasonic-absorbing porous coating is disposed on one half of the cone, which permitted to determine the transition position on the porous and solid surfaces in one experiment. Exchangeable nose tips with bluntness radii $R=0$, $1,2,4$, and $8 \mathrm{~mm}$ are used. Heat-fluxes distributions are measured along the cone with calorimetric gages situated both on the porous and solid surfaces. The positions of the laminar-turbulent transition are determined from these data. Moreover, the heat-fluxes pulsations are measured by uncalibrated ALTP gages situated on the porous and solid surfaces.

It was found that the bluntness of the nose part increased effectively the length of the laminar flow. It was discovered that the sound-absorbing coating kept its efficiency at different bluntnesses of the cone nose part and increased effectively (1.3-1.85) the length of the laminar flow in each experiment. Measured pulsations of the heat flux showed that the sound-absorbing coating stabilized effectively the 2nd-mode disturbances.

\section{REFERENCES}

1. Malmuth, N. D., A. V. Fedorov, V. Shalaev, J. Cole, and A. Khokhlov. 1998. Problems in high speed flow prediction relevant to control. AIAA Paper No. 98-2995,

2. Fedorov, A. V., N. D. Malmuth, A. Rasheed, and H. G. Hornung. 2001. Stabilization of hypersonic boundary layer by porous coatings. AIAA Paper No. 2001-0891.

3. Rasheed, A., H. G. Hornung, A. V. Fedorov, and N. D. Malmuth. 2001. Experiments on passive hypervelocity boundary layer control using a porous surface. AIAA Paper No. 2001-0274. 
4. Fomin, V., A. Fedorov, A. Shiplyuk, A. Maslov, E. Burov, and N. Malmuth. 2002. Stabilization of a hypersonic boundary layer by ultrasound-absorbing coatings. Doklady Phys. 47(5):401-4.

5. Maslov, A.A., A.N. Shiplyuk, A. A. Sidorenko, P. A. Polivanov, A. V. Fedorov, V. Kozlov, and N. D. Malmuth. 2006. Hypersonic laminar flow control using a porous coating. AIAA Paper No. 2006-1112.

6. Maslov, A. A., D. A. Bountin, A. N. Shiplyuk, B. V. Smorodsky, H. Knauss, U. Gaisbauer, S. Wagner, and J. Betz. 2004. ALTP sensor application for boundary layer measurements. Proc. Xth ICMAR. Part II. Novosibirsk, Russia. 137-46. 